\title{
Addressing Health-Related Myths in the Culturally Diverse African American Population: A Call to Action
}

Lucson Joseph

Nova Southeastern University, lucjoseph15@gmail.com

Follow this and additional works at: https://nsuworks.nova.edu/ijahsp

Part of the Bilingual, Multilingual, and Multicultural Education Commons, Community Health and Preventive Medicine Commons, Diseases Commons, Health and Physical Education Commons, Nutrition Commons, and the Public Health Education and Promotion Commons

\section{Recommended Citation}

Joseph L. Addressing Health-Related Myths in the Culturally Diverse African American Population: A Call to Action. The Internet Journal of Allied Health Sciences and Practice. 2021 Jan 01;19(3), Article 20.

This Opinion Piece is brought to you for free and open access by the College of Health Care Sciences at NSUWorks. It has been accepted for inclusion in Internet Journal of Allied Health Sciences and Practice by an authorized editor of NSUWorks. For more information, please contact nsuworks@nova.edu. 


\title{
Addressing Health-Related Myths in the Culturally Diverse African American Population: A Call to Action
}

\begin{abstract}
African Americans (AAs) experience numerous challenges that socially, economically, and physically affect their communities. Recent studies have found that a diverse array AAs encounter many struggles as they navigate the United States (U.S.) healthcare system to access care and receive healthcare services. AA communities are significantly affected by the burden of chronic diseases. They face considerable barriers to healthcare services that contribute to adverse health outcomes. This paper explains the daily struggles many AAs face within their communities to access and navigate the healthcare system due to culturally held myths and barriers. This paper discusses commonly held myths among Afro-Caribbean and West Indies populations living within the state of Georgia to suggest a call to action to address health disparities in this population.
\end{abstract}

\section{Author Bio(s)}

Lucson Joseph, DHSc, MPH, RRT is a vice-president for a non-profit organization that educates minorities about chronic disease management. He is an Infection Preventionist and a licensed Respiratory Care practitioner in the state of Georgia.

\section{Acknowledgements}

I would like to acknowledge Dr. Rachele Hendricks-Sturrup for her contribution and the time and effort put forth to help me write this paper. 


\title{
IJAHSP \\ The Internet Journal of Allied Health Sciences and Practice Vol. 19 No. 3 ISSN 1540-580X
}

\section{Addressing Health-Related Myths in the Culturally Diverse African American Population: A Call to Action}

\author{
Lucson Joseph \\ Nova Southeastern University \\ United States
}

\begin{abstract}
African Americans experience numerous challenges that socially, economically, and physically affect their communities. Recent studies have found that African Americans encounter many struggles to navigate the U.S. healthcare system to access care and receive healthcare services. African American communities are significantly affected by the burden of chronic diseases. They face considerable barriers to healthcare services that contribute to adverse health outcomes. This paper explains the daily struggles many African Americans face in their communities to access and navigate the healthcare system due to myths and cultural barriers. The myths among different African American groups, such as Afro-Caribbean and West Indies populations, are underlined in this paper to suggest a call to action for addressing health disparities in diverse African American communities in the U.S. (particularly in the state of Georgia).
\end{abstract}

Keywords: Chronic disease myths, culture, African American health, diverse communities, disease myths, health disparities, community health, cultural barrier, cultural myths 


\section{INTRODUCTION}

The African American population in the United States (US) consists of a diverse population of African descent and a subgroup of foreign-born immigrants. ${ }^{1}$ The Office of Minority Health (OMH) estimated that approximately $10 \%$ of the African American (AA) population in the US migrated from the Caribbean and Latin America. ${ }^{1}$ As a group, the AA community encounters substantial disparities with chronic diseases and access to health care services. AAs face considerable barriers that socially, economically, and physically affect their communities, contributing to poor health outcomes due to cultural myths and misconceptions. However, research has not captured the full spectrum of these diverse health outcomes. Although African descent populations are often vaguely classified under a single label, such as Black, numerous studies have shown that significant differences exist in health status among different AA populations. Every AA-ethnic group has a unique culture and behavior; each lives in a milieu that aggregates social and cultural conditions that influence their health. African descendants are notable in terms of culture, beliefs, behaviors, and risk factors, such as height, weight, and disease experience. ${ }^{2}$ It is reported that AAs are 80 percent more likely to be diagnosed with diabetes and 2.4 times more likely to initiate treatment for end-stage renal disease. ${ }^{3}$ Also, AAs are 1.7 times more likely to be hospitalized due to diabetes complications and more likely to have a visual impairment. ${ }^{3}$

The prevalence of heart disease is also high in the AA community. The Center for Disease Control and Prevention (CDC) 3 reported that AA men are 30 percent and women are 60 percent more likely to have high blood pressure, and are even less likely to keep blood pressure under control. In addition, the CDC noted that AAs have twice the risk of a first-time stroke. ${ }^{3}$ Regarding cancer prevalence and rate, AA women are 40 percent more likely to die of breast cancer, and AA males are more likely to be diagnosed with colorectal cancer. ${ }^{3}$ Among other chronic issues, maternal and child health are priorities in the AA community. AA children are 1.8 times more likely to have asthma and are 3.5 times likely to die from low-birthweight-related complications. ${ }^{3}$ These disparities are more granular at the local levels. The state of Georgia is a crucial example where AAs encounter significant health disparities across this same spectrum relative to the general population.

In a recent report, the Georgia Department of Public Health indicated that chronic diseases, such as heart disease, cancer, diabetes, and chronic respiratory disease, are the leading causes of death and disability in Georgia. ${ }^{4}$ The CDC asserted that AAs are 40 percent more likely to have a higher blood pressure than whites and are less likely to have the condition under control. ${ }^{2}$ Also, the Georgia Department of Public (GDPH) reported that Georgia's stroke mortality rate is greater than the U.S., and the rate is significantly higher for blacks than whites. ${ }^{4}$ According to the National for Health Statistics, diabetes rates are $77 \%$ higher among AAs than whites, and life expectancy is nearly four years less. ${ }^{5}$ More than one million Georgians (14\%) have been diagnosed with diabetes, and a significant percentage are pre-diabetic (36.1\%). ${ }^{4,6} \mathrm{It}$ is important to note that chronic disease disparity is significantly higher in the underserved urban and rural Georgia counties. One county that is profoundly affected by the chronic disease crisis in Georgia is Dekalb County. According to the U.S. Census Bureau, Dekalb County Georgia consists of more than 700,000 people and is the third largest and the most culturally diverse county in Georgia. ${ }^{6,7} \mathrm{It}$ is estimated that $16 \%$ of Dekalb county residents are foreign-born, and $18 \%$ speak a language other than English at home. ${ }^{7,8} \mathrm{It}$ is important to underline that there are numerous ethnic groups and cultural nuances within the African American populations. Thus, innumerable gaps exist in access to evidence-based intervention that could reduce chronic diseases in diverse underserved areas in the state of Georgia.

\section{PUBLIC HEALTH CAUSES TO HEALTH DISPARITY IN THE AA COMMUNITY IN GEORGIA}

There are numerous public health causes related to health disparities in the AA communities in Georgia and the U.S. that are noteworthy. First, the AA community consists of diverse people from Africa, the West Indies, and Latin America. These groups have unique cultural values, which are often different in terms of language, diet, beliefs, and migration history. Thus, health outcomes among diverse populations are expected to be different, which underlines the need for more epidemiological research in public health. In addition, care inaccessibility impedes AA communities across all groups and ages. It is a well-known fact that access to comprehensive and quality healthcare services is essential for an individual's wellbeing. Nevertheless, AAs of all demographics and ages continue to face significant barriers to health care access. For example, the Georgia Center for Opportunity $(\mathrm{GCO})^{9}$ estimated that about 19 percent of Georgians lack health insurance, representing the country's sixth-highest percentage in 2012. Also, among nonelderly uninsured, about 56 percent live in households that earn less than 139 percent of the federal poverty level (FPL), which is $\$ 16,221$ for an individual and $\$ 33,151$ for a family of four. ${ }^{9}$ While many uninsured Georgians use community health centers, charity clinics, public hospitals, local health departments, and private office limited free care services, there are significant health care needs that remain unmet. ${ }^{9}$ Lack of access to affordable care contributes to many individuals going without essential services. In addition, uninsured Georgians are nearly four times less likely than the insured to have a routine yearly check-up and more likely to experience a preventable hospitalization for health conditions, such as pneumonia, diabetes, and asthma. ${ }^{9}$ 
In addition to health care access, food deserts are another issue that is hampering many underserved minority communities in Georgia. When it comes to making nutritional foods accessible to all Georgians, food deserts are considered a significant problem in Georgia. Staples explained in a recent report that nearly 2 million Georgians, including 500,000 children, live in food desert areas. ${ }^{10}$ The USDA has classified more than 35 food desert areas inside the metro Perimeter, including the border of I-285 in the suburbs of Cobb, South Fulton, and East Dekalb counties. ${ }^{10}$ The severity of the food deserts situation in the Metropolitan area led many to believe there is a direct correlation between food deserts and the state's high rates of obesity and chronic diseases. ${ }^{10}$ In the state of Georgia, heart disease and stroke are among the top three leading causes of death, accounting for nearly one-third of all deaths in the state. $5,10,11$

\section{CURRENT HEALTH-RELATED MYTHS AMONG CULTURALLY DIVERSE AFRICAN AMERICAN POPULATION}

As the U.S. population becomes more racially and ethnically diverse, it is vital to grasp the importance of addressing health disparity at a different angle to meet the culturally diverse AA population's needs. Caballero ${ }^{13}$ described several health-related myths and misconceptions about the management of obesity, diabetes, heart disease, and cancer among AAs. ${ }^{13}$ For example, for many foreign-born AAs, obesity is a sign or expression of financial and social status, prosperity, and reflects access to adequate amounts of food and indicates a good life. ${ }^{13}$ Regarding diabetes, a common myth among foreign-born AAs is that diabetes is not a severe medical condition that requires diet modification. ${ }^{14,15}$ Although these myths and misconceptions might be grounded in tradition or culture or hold validity in undocumented contexts, they can inevitably cultivate serious health consequences, such as morbidity and mortality. 10,14 Evidently, the high prevalence rate of chronic diseases and mortality rate in the AA community is multifaceted. As a result, diabetes-related complications have been reported to decrease life quality among these groups because of a lack of proper management. Interestingly, these myths are not only found in Georgia or Dekalb County. These myths are found across the US in disproportionately over-populated areas with African decedents, particularly Afro-Caribbean decedents. ${ }^{14,15}$ The AfroCaribbean and West Indies share a remarkable similarity in their health behaviors, and those behaviors are not restricted to geographical location, age, or gender. ${ }^{14,16}$ There are numerous myths about chronic diseases that are commonly reported. Some of the related myths include:

1. Eating a high amount of sugar can cause diabetes.

2. Prayer can cure diabetes and hypertension.

3. Heart disease is mainly found in men.

4. Taking natural supplements can cure heart disease.

5. Naturopathic medicine is more useful than insulin treatment for diabetes.

6. Diabetes manifests only within the elderly.

7. Diabetes treatment should be ceased if it can be controlled with herbal medicine.

8. Insulin means he/he fails to manage diabetes.

9. Unsalted food can cure hypertension.

Consequently, fewer public health programs aim to decipher the root causes and influential factors across cultural differences to solve these myths. Some of the factors that lead to this enormous public health crisis are environmental and/or neighborhood sways, racial inequality, health behaviors, diet, and cultural perception of health care among diverse communities. ${ }^{14}$ Similar myths and misconceptions related to diabetes, heart disease, and cancer can also quickly grow and spread in the AA community. One striking example is the COVID-19 pandemic, which revealed how the AA population could promptly become susceptible to unfounded health-related rumors and myths. ${ }^{17}$ In early March of 2020, when the virus began spreading across the country, several myths and misconceptions were circulating in the AA community about "Black immunity" to the Coronavirus on social media. ${ }^{18}$ While the concepts behind "Black immunity" inspired the perception that African descendants' immune response significantly differed, especially in genes involved in inflammatory and antiviral responses. However, early infection-rate reports showed the contrary. Johnson and Buford reported that AAs were contracting Coronavirus at a relatively higher rate and were more likely to be hospitalized or die from the virus due to comorbidities, such as diabetes, asthma, and heart disease. ${ }^{18}$ According to the Georgia Department of Public Health, a recent survey conducted by the CDC in Atlanta reported that more than 80 percent of hospitalized coronavirus-infected patients in Georgia were AAs. ${ }^{20}$ Other reports from Chicago and New Orleans suggested that 70 to 80 percent of Covid-19 cases were AAs. ${ }^{17,19}$ The virus' rapid death rates across the nation expose the extent to which AAs are adversely affected by the pandemic as a result of pre-existing cardiovascular disease, diabetes, obesity, socioeconomic factors, and increased likelihood of occupational coronavirus exposure. ${ }^{17,18,19}$

While there is no evidence to support that AAs have any genetic pre-disposition types that make them more likely to be affected by COVID-19, AAs are more likely to have an underlying health condition. Chronic conditions, such as diabetes, heart disease, obesity, and asthma, are very prominent in the AA Community. ${ }^{20}$ These conditions increase the risk of severe complications with 
COVID-19.20 Williams and Cooper explained that COVID-19 is a "magnifying glass highlighting the larger pandemic of racial/ethnic disparities in health across the US." 21 Also, Williams and Cooper asserted that higher morbidity and mortality from chronic diseases tend to exist in diverse racial and ethnic minority communities for many unknown reasons. ${ }^{21}$ In fact, among older adults, a higher proportion of AAs and Latinos reported having at least one of the eight chronic conditions, including asthma, cancer, heart disease, diabetes, high blood pressure, obesity, anxiety, or depression. ${ }^{18}$ In addition, Laurencin and McClinton asserted that social inequities in America are determined by numerous factors, while the opportunities to stay healthy vary plainly at the neighborhood level. ${ }^{19}$ Thus, the influence of cultural myths in the AA communities demonstrates that less emphasis has been placed on addressing several essential public health elements, including cultural determinants of health in diverse AA populations. ${ }^{21,22}$ The Covid-19 pandemic reveals that less emphasis has been placed on addressing the educational, social, financial, psychological, and cultural determinants of health and disease in culturally-diverse AA populations. ${ }^{20,21,22}$

\section{DISCUSSION}

Given the recent surge of migration in the past decades, cultural nuances have become a more critical issue than ever for public health officials in the AA population. The combination of Afro-Caribbean descent, West Indies, Africans, and Latin Americans descents living in the U.S. makes the AA population one of the most unique and diverse populations. While many studies group these ethnically diverse populations as Black, remarkably, each ethnic group has its distinctive cultural traits and nuances. Some racial groups present substantial health challenges to the U.S. health care system and often require extra resources to address. For example, Afro-Caribbean and West Indies descendants have different ways of thinking about healthcare and may have traditions and conflicting views against conventional Western medicine. ${ }^{23}$ These cultural assumptions can create a unique challenge for both healthcare professionals and patients. Thus, healthcare providers who are not familiar with cultural traditions surrounding medical care may have difficulty connecting with these patients. Also, the understanding of healthcare services can be varied from culture to culture. Therefore, healthcare professionals, alongside public health officials, should ensure that patients are educated and understand their diagnosis and treatments in a sensitive way to their cultural needs.

Undoubtedly, addressing health disparities in the AA community means tackling existing and contemplating developments that can inspire health-related myths, poor health behavior, and outcomes in culturally diverse AA populations. Implementing appropriate interventions to dispel health-related myths among culturally diverse AA populations is also essential in addressing health disparities and equity. Public health officials and healthcare providers must examine, understand, and embrace diversity in a broader context to deliver health care services and implement programs in a culturally oriented manner. Numerous social determinant elements affect diverse culturally oriented communities, and these factors must be understood to address health disparity appropriately. For example, in culturally diverse AA communities, religion, cultural beliefs, and values play a significant role in managing and curing disease, particularly in managing chronic diseases, such as diabetes, hypertension, and cancer. ${ }^{16,23}$ The lack of culturally and linguistically available resources, proper health insurance coverage, and the lack of understanding of the U.S. healthcare system are essential issues that must be resolved among diverse AA communities. ${ }^{23}$

\section{A CALL TO ACTION}

Evidently, the underlying causes of the health disparities in culturally diverse AA communities can be linked to genetics, socioeconomic or economic status, limited access to health care or insurance status, delays in treatment, cultural and religious beliefs, low literacy and/or health literacy rates, as well as certain environmental factors. ${ }^{21,23,24}$ These factors can be perplexing to address and can interrupt the delivery of healthcare services to culturally diverse populations. Although considerable progress has been made over the years to narrow the gap in health outcomes, the elimination of health disparities in culturally diverse communities has yet to be accomplished. ${ }^{23,24}$ Lewis and Steinert asserted that culture should be used as a foundation to develop effective health and human services prevention, treatment, and the allocation of health care services in culturally diverse communities. ${ }^{25}$ Thus, addressing health disparities in the AA community is to challenge the belief system and the AAs' existing mindset. ${ }^{26}$ Health inequities across the United States are well-documented. ${ }^{28}$ Therefore, providing culturally responsive treatment and services could bring awareness of cultural attitudes, beliefs, biases, and health assumptions that co-exist in culturally-diverse populations. ${ }^{27,28,29}$

Numerous studies suggest tremendous variation exists in disease among racial/ethnic groups in the United States. Local data from state to state tend to magnify those differences and the need for intervention to address these pervasive health issues among different population groups. Consequently, understanding the nuances and barriers facing culturally diverse AA populations is critical to improving life quality and disease management among these communities. I call for state and public health officials to develop strategies that address the complexity of health disparities among culturally diverse AA populations, thereby addressing the myths associated with relatively higher disease prevalence and poorer disease outcomes in those communities. Also, healthcare professionals and public health officials should be trained on cultural sensitivity to develop innovative ways for 
addressing the burdens of disease, poverty, limited health care access, and culture. Lastly, it is critical to address health disparities in a cultural context to respond to specific challenges facing the multi-cultural AA population living in the US.

\section{CONCLUSIONS}

The number of ethnically and culturally diverse groups in the United States is rapidly growing. Interestingly, each group has its own and unique cultural traits. It has been noted that some of these racial groups have significant challenges in accessing the U.S. health care system due to cultural myths and misconceptions. Thus, addressing health disparities in the AA community means tackling existing and contemplating developments that can inspire health-related myths, poor health behavior, and outcomes. Numerous health elements affect culturally oriented communities, including the lack of health literacy and equity. These elements are often associated with many health complications and deaths in culturally oriented populations. In those communities, religion, cultural beliefs, and values play a significant role in managing and treating disease, particularly managing chronic conditions like diabetes, hypertension, and obesity. These factors must be understood to address health disparity appropriately. Therefore, there is a considerable need for appropriate interventions to dispel health-related myths while addressing health disparities and equity in culturally diverse AA populations.

\section{REFERENCES}

1. Perez AD, Hirschman $C$. The Changing Racial and Ethnic Composition of the US Population: Emerging American Identities. Popul Dev Rev. 2009; 35(1):1-51. doi:10.1111/j.1728-4457.2009.00260.x. Accessed November 24, 2020

2. Davis, I. J., Brown, C. P., Allen, F., Davis, T., \& Waldron, D. African-American myths and health care: The sociocultural theory, 1995. Journal of the National Medical Association, 87(11), 791-794. Access March 05, 2020

3. Centers for Disease Control and Prevention. The four domains of chronic disease prevention, 2015. https://www.cdc.gov/chronicdisease/resources/publications/four-domains.htm. Access March 03, 2020.

4. Center for Disease Control and Preventions. Health of black or African Americans non-Hispanic population, 2017. https://www.cdc.gov/nchs/fastats/black-health.htm. Accessed March 03, 2020.

5. Georgia Department of Public Health. Chronic Disease Prevention, 2017. https://dph.georgia.gov/chronic-diseaseprevention. Accessed January 21, 2020.

6. National Center for Health Statistics. Health, United States, 2018. Published 2019. https://www.cdc.gov/nchs/data/hus/hus18.pdf. Accessed May 7, 2020

7. Rollins, L., Akintobi, T. H., Hermstad, A., Cooper, D., Goodin, L., Beane, J., Spivey, S., Riedesel, A., Taylor, L., \& Lyn, R. Community-based approaches to reduce chronic disease disparities in Georgia, 2017. J Ga Public Health Assoc, (6)4. Accessed April 10, 2020.

8. US Census Bureau. Quick Facts, DeKalb County, Georgia, 2017. https://www.census.gov/quickfacts/table/PST045215/13089,00. Accessed March 7, 2020.

9. Intergenerational poverty project. Georgia Center for Opportunity. Qualitative Online Survey Report, 2018. https://georgiaopportunity.org/intergenerational-poverty-study/Accessed October 19, 2020

10. Staples, G. B. Poor diets and inaccessibility to healthy foods are creating a crisis of chronic disease, 2016. Starving for nutrition. The Atlanta Journal-Constitution. https://investigations.ajc.com/fooddeserts/. Accessed October 28, 2020

11. DeKalb County Board of Health. 2015 DeKalb County Status of Health Report, 2015. https://dekalbhealth.net/wpcontent/uploads/2016/02/SOH-2015-FINAL-Web-Version.pdf. Accessed April 19, 2020

12. American Nutrition Association. USDA Defines Food Deserts, 2017. http://americannutritionassociation.org/newsletter/usda-defines-food-deserts. Accessed April 13, 2020.

13. Caballero, A. E. The "A to Z" of managing type 2 diabetes in culturally diverse populations, 2018. Frontier in Endocrinology. 2018; 9(447). doi: 10.3389/fendo.2018.00479. Accessed March 17, 2020

14. Bennett, N. R., Bidulescu, A., Ferguson, T. S., Francis, D. K., Hambleton, I. R., Harris, E. N., Hassell, C., Hennis, A. J. M., MacLeish, M.Y., Sullivan, L.W., Wilks, R. J., Williams, L., \& Younger-Coleman, N. O. Disparities in diabetes mellitus among Caribbean populations: A scoping review, 2015. International Journal Equity Health, 14(1), 1-17. https//doi.org/10.1186/s12939-015-0149-z. Accessed November 16, 2020

15. Agyemang C, Bhopal R, Bruijnzeels M. Negro, Black, Black African, African Caribbean, African American or what? Labelling African origin populations in the health arena in the 21st century, 2005. Journal of Epidemiology \& Community Health, 59, 1014-1018. Accessed November, 272020

16. Butler A. M. Social determinants of health and racial/ethnic disparities in type 2 diabetes in youth, 2017. Current Diabetes Reports, 17(8), 60. https://doi.org/10.1007/s11892-017-0885-0 
17. Jones, V. Black American must wake up to this viral threat, 2020. CNN Opinion. Published 2020. https://www.cnn.com/2020/04/06/opinions/african-americans-covid-19-risk-jones/index.html. Accessed May 10, 2020.

18. Johnson, A., Buford T. Early data shows African Americans have contracted and died of Coronavirus at an alarming rate, 2020. ProPublica. Published 2020. https://www.propublica.org/article/early-data-shows-african-americans-havecontracted-and-died-of-coronavirus-at-an-alarming-rate. Accessed May 9, 2020.

19. Laurencin, C. T., \& McClinton, A. The COVID-19 Pandemic: a Call to Action to Identify and Address Racial and Ethnic Disparities, 2020. Journal of racial and ethnic health disparities, 7(3), 398-402. https://doi.org/10.1007/s40615-02000756-0. Accessed November 15, 2020

20. Georgia Department of Public Health. Georgia overall Covid-19 Status, 2020. Published 2020. https://dph.georgia.gov/covid-19-daily-status-report. Access May 02. 2020.

21. Williams DR, Cooper, LA. COVID-19 and Health Equity-A New Kind of "Herd Immunity". JAMA. Published online May 11, 2020. doi:10.1001/jama.2020.8051. Accessed May 13, 2020.

22. John Hopkins University. Coronavirus resource center, 2020. https://coronavirus.jhu.edu/map.html. Accessed April 28, 2020.

23. Butler, M., McCreedy, E., Schwer, N., Burgess, D., Call, K., Przedworski, J., Rosser, S., Larson, S., Allen, M., Fu, S., \& Kane, R. L. Improving cultural competence to reduce health disparities. Agency for healthcare research and quality, 2016. Comparative Effectiveness Reviews, 170. https://www.ncbi.nlm.nih.gov/books/NBK361126/. Accessed November 20, 2020

24. Krieger, N., Singh, N., Chen, J. T., Coull, B. A., Beckfield, J., Kiang, M. V., Waterman, P. D., \& Gruskin, S. Why history matters for quantitative target setting: Long-term trends in socioeconomic and racial/ethnic inequities in US infant death rates (1960-2010), 2015. Journal of Public Health policy, 36(3), 287-303. https://doi.org/10.1057/jphp.2015.12. Accessed November 10, 2020

25. Lewis, L. D., \& Steinert, Y. How culture Is understood in faculty development in the health professions: A scoping review, 2020. Academic Medicine: Journal of the Association of American Medical Colleges, 95(2), 310-319. https://doi.org/10.1097/ACM.0000000000003024. Accessed November 13, 2020.

26. Center for Disease Control and Preventions. About chronic diseases. National Center for Chronic Disease Prevention and Health Promotions, 2018. https://www.cdc.gov/chronicdisease/about/index.htm. Accessed March 15, 2020.

27. Jongen, C., McCalman, J., \& Bainbridge, R. Health workforce cultural competency interventions: A systematic scoping review, 2018. BMC Health Services Research, 18(1), 232. https://doi.org/10.1186/s12913-018-3001-5

28. McCalman, J., Jongen, C., \& Bainbridge, R. Organisational systems' approaches to improving cultural competence in healthcare: A systematic scoping review of the literature, 2017. International Journal for Equity in Health, 16(1), 78. https://doi.org/10.1186/s12939-017-0571-5. Accessed November 22, 2020.

29. Office of Minority Health. (2019). Diabetes and African Americans, 2019. https://minorityhealth.hhs.gov/omh/browse.aspx?lvl=4\&lvlid=18. October 17, 2020 